\title{
Radiometric Location of Partial Discharge Sources for the Future Smart Grid
}

\author{
José Mauricio Neto ${ }^{1}$, Yong Zhang ${ }^{2}$, Adel Jaber ${ }^{2}$, M Zhu ${ }^{3}$, Martin Judd ${ }^{3}$, Robert Atkinson ${ }^{3}$, John Soraghan ${ }^{3}$, José $^{\prime}$ \\ Sergio Neto ${ }^{1}$, Maria de Fatima $Q$ Vieira ${ }^{1}$ and Ian A Glover ${ }^{2}$ \\ ${ }^{1}$ Department of Electrical Engineering, Federal University of Campina Grande, Campina Grande, Brazil, \\ jose.neto@ee.ufcg.edu.br \\ ${ }^{2}$ Department of Engineering \& Technology, University of Huddersfield, Huddersfield, UK \\ y.zhang@hud.ac.uk \\ ${ }^{3}$ Department of Electronic \& Electrical Engineering, University of Strathclyde, Glasgow, UK \\ m.judd@strath.ac.uk
}

\begin{abstract}
Measurements are reported that suggest partial discharge sources might be detected and located using amplitude-only free-space radiometric measurements. The measurements are made in two locations with similar measurement protocols by three collaborating research groups. One measurement set is more encouraging than the other but a likely explanation for the disparate nature of the results is offered. It is suggested that amplitude only measurements might form the basis of a low-cost wireless sensor network for monitoring partial discharge in electricity substation.
\end{abstract}

\section{Introduction}

Partial discharge (PD) refers to a discharge that does not completely span the electrodes producing it. Fig. 1 illustrates the origin of PD in an insulator containing a void subjected to a high voltage. Since the void has lower

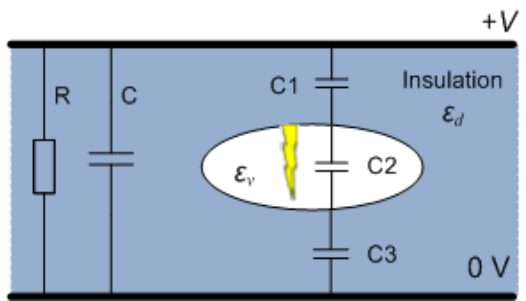

Figure 1. Origin, and equivalent circuit of partial discharge electrical permittivity than the surrounding insulation $\left(\varepsilon_{v}<\varepsilon_{d}\right)$ the electric field strength within the void is greater than that in the surrounding insulation. Furthermore, the insulation strength of the void is less than that of the insulation. Both these factors mean that the electric field may exceed the breakdown value inside the void without exceeding the breakdown value in the insulation resulting in a discharge in the void but not in the surrounding dielectric. When such a discharge occurs, the electric field within the void collapses and so the discharge is extinguished. Assuming the potential difference across the electrodes persists, the electric field across the void then rises again initiating a subsequent discharge. PD is therefore characterised by a series of discrete current pulses. The duration of these current pulses for dielectrics typically used as insulation in power systems equipment is measured on a timescale of nanoseconds to microseconds. The spectrum of PD can therefore extend into the gigahertz region although most of the energy usually resides below a few hundred megahertz.

PD detection systems can be broadly divided into electrical contact, near-field coupling, internal UHF and external UHF (referred to here as free-space RF) methods [1, 2]. The electrical contact methods (e.g. as described in IEC60270) are both sensitive and accurate but require plant to be disconnected from the power system and energized by an external voltage source. Near-field coupling methods, which include high frequency current transformers (HFCTs) and transient earth voltage (TEV) sensors, require only physical (rather than electrical) connection to the item of plant being monitored. Internal UHF methods involve the installation of a UHF antenna inside an item of plant (often a large transformer in which case the antenna is typically installed via the oil drainage valve). This paper addresses free-space RF detection of PD which is less easily calibrated than other methods but has the advantages that no physical contact is needed with any item of plant and fewer sensors are needed to monitor an entire substation. A monitoring system using free-space RF sensors is also easier to install and reconfigure than any of the other methods.

\section{Free-space RF measurements for PD source location}

Free-space RF PD source location has been successfully implemented using time-difference-of-arrival (TDOA) measurements $[2,3]$. This involves a modest number $(>3)$ of spatially separated antennas connected to receivers with a sufficiently high sampling rate to record the received RF signals of PD origin. The signals are cross-correlated to yield the TDOA of the PD signal at each antenna and these TDOAs are used to estimate the location of the PD source.

A more economical method of PD monitoring and location might be to employ PD intensity measurements followed by source location using, for example, path-loss law inversion [4]. Such measurements could be gathered using a broadband receiver as the sensing element at each node of a conventional wireless sensor 
network (WSN). Since the future Intelligent (or Smart) Grid will almost certainly incorporate one or more WSNs to monitor many other power systems quantities of interest the WSN infrastructure required for PD monitoring may come at little, if any, marginal cost. The WSN technology is likely to operate at $2.45 \mathrm{GHz}$ ISM band, well above the frequency band containing the majority of PD energy (typically $50-800 \mathrm{MHz}$ ) to avoid performance degradation due to interference of PD origin. Using this technology will also ensure that WSN communication signals do not inflate PD intensity measurements if intensity is determined by a gross power measurement.

\subsection{PD intensity measurement}

Although PD occurs as a series of narrow current pulses, the signal received by a free-space RF sensor is typically characterized by a decaying, noisy, oscillation, Fig. 2 (upper trace). This is because the received signal
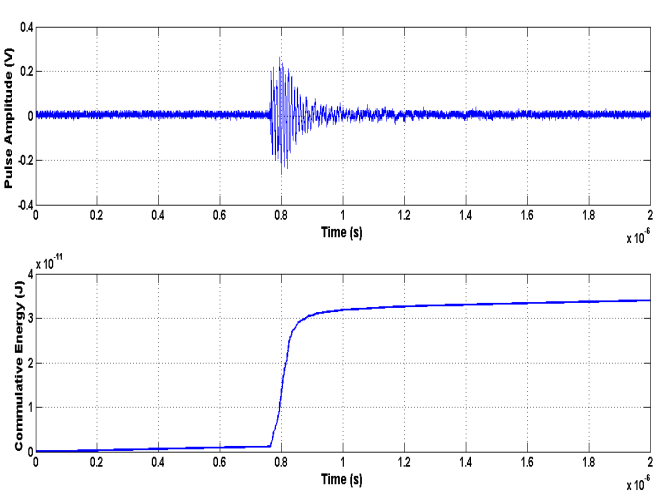

Figure 2. Received RF signal due to floatingelectrode PD (upper trace) and cumulative energy (lower trace) is essentially the impulse response of the band-limited system formed by the PD radiating structure, the wireless channel and the receiver (including the receive antenna). The peak amplitude of the received RF signal pulse may not be the most reliable metric of PD intensity. The calculation of cumulative $\mathrm{RF}$ energy as suggested in [1] has the advantage of using the entire pulse and reducing noise, Fig. 2 (lower trace).

\subsection{Location algorithm}

A PD source lying close to one WSN node will have decayed to relatively low levels at the locations of more distant nodes. Interference, including narrowband communications, lying within the PD signal measurement band but originating from outside the substation compound, will generally be far from all nodes and will therefore 'bias' the intensity measurements of all nodes equally. Radiometer 'hot-spots' superimposed on a map of the substation will therefore reflect signals of substation origin only. An approximately uniform spatial distribution of nodes will thus give the network an inherent PD localization capacity. If the radio propagation path-loss index is known (or can be estimated) then localization can be refined by considering the energies measured at pairs of nodes. The ratio of energies at a pair of nodes together with the path-loss index implies a ratio of distances from the nodes to the source which defines a locus on which the source lies. A PD source detected by three or more radiometers can therefore be located (in a horizontal plane) from the intersection of two or more loci. (With more nodes then the source's height can also be located and location ambiguities due to multiple loci intersections be resolved.)

\subsection{An alternative signal processing approach}

The direct calculation of cumulative signal energy requires large amounts of high-frequency data to be captured using a high-speed ADC. Envelope detection may offer significant potential for sampling rate reduction at the expense of a modest performance penalty, allowing signals to be sampled, digitized and stored using more economical hardware [5] and a reduction in the energy required for signal processing. Reduced energy requirements will lead to longer battery life for the WSN nodes and/or the possibility of powering nodes indefinitely using energy harvesting technologies.

\section{Measurements}

Proof of principle measurements have been made by three collaborating research groups in two laboratories resulting in two datasets. Dataset 1 represents measurements made in the HV laboratory of the Department of Electronic \& Electrical Engineering at the University of Strathclyde. Dataset 2 represents measurements made in the Power Engineering laboratory of the Department of Engineering \& Technology at the University of Huddersfield. Fig. 3 shows the measurement spaces and measurement geometries.

PD pulses were generated using the floating-electrode test cell shown in Fig. 4(a). A DC voltage applied to the cell and increased until PD started to occur. The minimum voltage resulting in PD radiation was $13 \mathrm{kV}$. An emulated PD sensor was used to record the received PD signal. The sensor was moved sequentially between locations to gather the data that would be collected by an ensemble of sensors. There was no change in the laboratory environment between measurements.

A difference between these measurements and an operational system is that the PD signals collected at each spatial location represent different instances of PD generated by the test cell rather than data generated by common PD pulses. (This may not be unrealistic, however, since if the sensors are not explicitly timesynchronized then identifying received pulses originating from a common radiated pulse may not be practical.) 


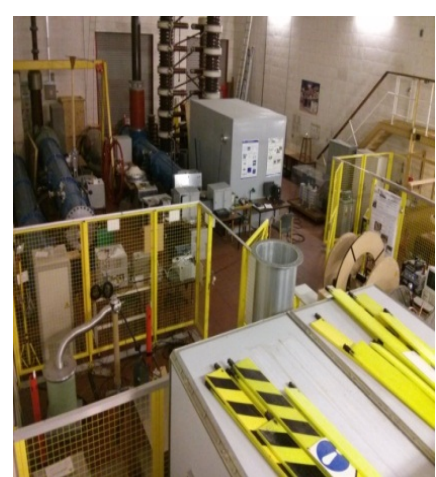

(a)

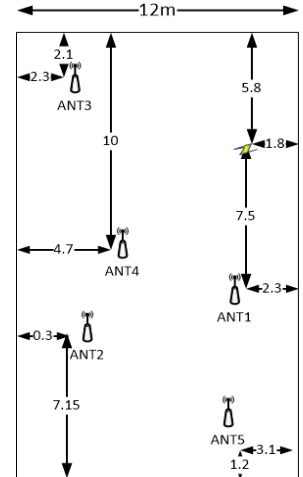

(b)

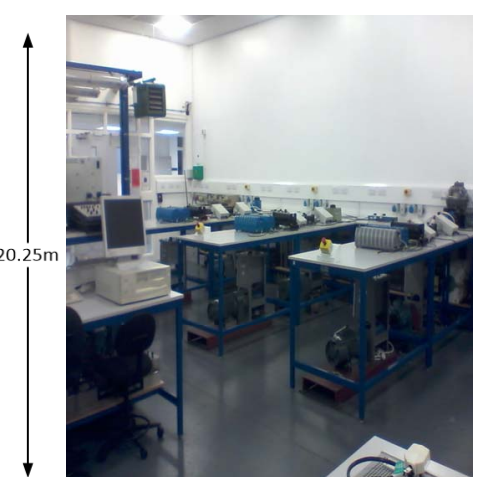

(c)

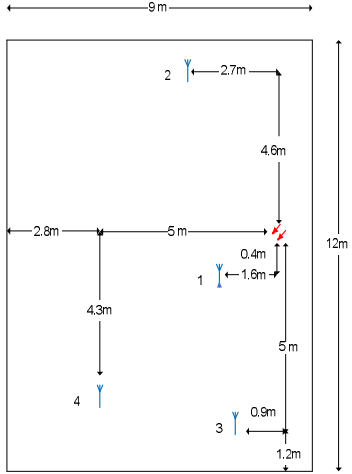

(d)

Figure 3 (a) Strathclyde measurement space (HV laboratory, dataset 1), (b) Measurement geometry for dataset 1, (c) Huddersfield measurement space (Power Engineering laboratory, dataset 2), (d) Measurement geometry for dataset 2

Two sensor types were emulated for each measurement. Sensor 1 comprised a UWB omnidirectional antenna, RF low noise amplifier (LNA) and high-bandwidth digital storage oscilloscope (DSO). Sensor 2 incorporated an envelope detector, DC blocking capacitor, low-pass (smoothing) filter and operational amplifier impedance buffer. Fig. 4(b) is a block diagram of the emulated sensors. The specification of the instrumentation for both datasets is summarized in Table 1.

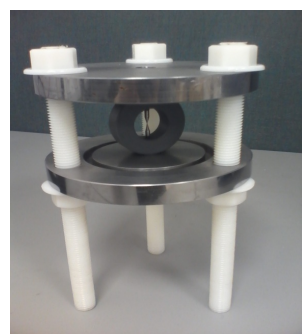

(a)

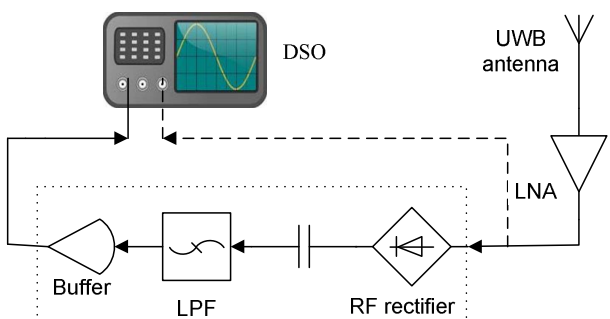

(b)

Figure 4 (a) Floating-electrode PD emulator and (b) Emulated sensors

The location of the PD source was estimated using both the RF signals and the envelope-detected signals. In both cases the energies of 100 received PD pulses were calculated at each emulated sensor node position. The mean value of the energy at each location was then used to estimate the location of the PD source using the algorithm described in Section 2.2.

Table 1. Emulated sensor parameters

\begin{tabular}{|l|c|c|}
\hline & Dataset 1 & Dataset 2 \\
\hline Laboratory dimensions & $20 \mathrm{~m} \times 12 \mathrm{~m}$ & $12 \mathrm{~m} \times 9 \mathrm{~m}$ \\
\hline Antenna (bandwidth) & Disk-cone $(200 \mathrm{MHz}-1 \mathrm{GHz})$ & Biconical $(20 \mathrm{MHz}-1 \mathrm{GHz})$ \\
\hline RF amplifier gain & None & $16 \mathrm{~dB}$ \\
\hline RF amplifier noise figure & None & $3 \mathrm{MB}$ \\
\hline Envelope detector bandwidth & $3 \mathrm{MHz}$ & $10 \mu \mathrm{F}$ \\
\hline DC blocking capacitor & $10 \mu \mathrm{F}$ & $3 \mathrm{MHz}$ \\
\hline Low pass filter bandwidth & $3 \mathrm{MHz}$ & $0 \mathrm{~dB}$ \\
\hline Operational amplifier (impedance buffer) & $0 \mathrm{~dB}$ & $20 \mathrm{GSa} / \mathrm{s}$ \\
\hline Oscilloscope sampling (RF signal) & $5 \mathrm{GSa} / \mathrm{s}$ & $10 \mathrm{MSa} / \mathrm{s}$ \\
\hline Oscilloscope sampling (envelope detection) & $10 \mathrm{MSa} / \mathrm{s}$ & 4 \\
\hline No. of measurement locations & 5 & \\
\hline
\end{tabular}

\section{Results}

Fig. 5 shows the estimated PD source locations (red crosses) for both datasets and energies calculated from both RF signal and envelope detected signal. Each location estimate corresponds to a different assumed value of path-loss index $n$ between 1 and 7. In an operational system the path-loss index may be estimated from a TDM calibration signal transmitted (with very small duty cycle) from each sensor node to all other sensor nodes. Alternatively it may be possible to use an iterative location algorithm in which path-loss index is adjusted in 
small steps until the RMS spread of loci intersections is minimized. Table 2 shows the errors between estimated and actual PD source locations for a path-loss index, $n$, of 2 .

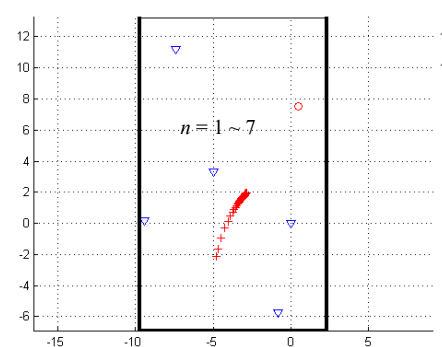

(a)

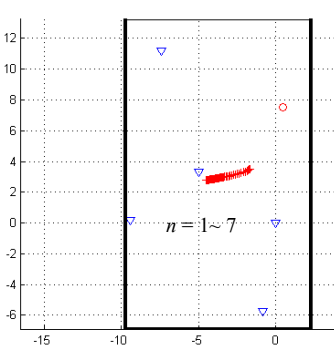

(b)

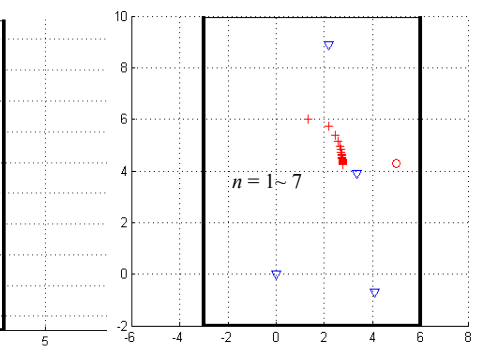

(c)

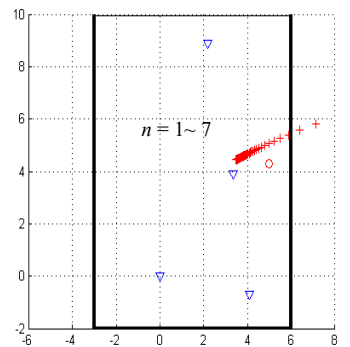

(d)

Figure 5. Location estimates: (a) dataset 1 RF signals, (b) dataset 1 envelope detected signals, (c) dataset 2 RF signals, (d) dataset 2 envelope detected signals. Key: red circle - actual PD source location, blue triangles - emulated measurement node locations, red crosses - PD source location estimates. (Axes measured in metres.)

Table 2. Location errors for $n=2$

\begin{tabular}{|l|l|l|}
\hline$n=2$ & RF signals & Envelope detected signal \\
\hline Dataset 1 & $6.89 \mathrm{~m}$ & $5.94 \mathrm{~m}$ \\
\hline Dataset 2 & $2.23 \mathrm{~m}$ & $0.93 \mathrm{~m}$ \\
\hline
\end{tabular}

The most striking feature of the results is the difference in source location error between the two datasets. The measurement environment for dataset 1 is more electrically complex than that for dataset 2, containing more electrically conducting clutter. Further investigation of the laboratory in which the measurements of dataset 1 were taken revealed that the floor, walls and ceiling contained earthed conducting mesh. The lab therefore effectively formed a Faraday cage. Consequently, the multipath structure of the field might be so intense that the use of a path-loss index to model transmission loss could be inappropriate.

The minimum location error is superior when using the envelope detector. This is surprising since the envelope detector uses half-wave rectification and thus decreases effective signal-to-noise ratio. Furthermore the envelope detection process can only increase distortion in the received signal, not reduce it. It is fortuitous, however, that the envelope detector appears to perform better because it has significant technological and cost benefits over RF energy detection.

\section{Conclusion}

Two laboratory-based investigations of the plausibility of using amplitude-only measurements to locate a PD source have been reported. The results are mixed; one dataset suggesting greater location accuracy is possible than the other. A possible explanation for the disparate location accuracies found is that dataset 1 is adversely affected by conducting mesh embedded in the floor, walls and ceiling of the laboratory in which the measurements were taken. Electricity substations, the application environment envisaged, will be multipath intensive but will not resemble the Faraday-cage character of this laboratory. The location errors found from the second dataset of measurements, whilst smaller than the first dataset, are probably still too large for the intended application. They are sufficiently encouraging, however, to warrant continued work.

Two types of detector have been used in the investigation: an RF energy detector and an envelope energy detector. The latter gave smaller location error than the former.

\section{Acknowledgment}

The authors acknowledge the support of the U.K. Engineering and Physical Sciences Research Council under Grant EP/J015873.

\section{References}

1. A. J. Reid, M. D. Judd, R. A. Fouracre, B. G. Stewart and D. M. Hepburn, "Simultaneous measurement of partial discharges using IEC60270 and radio-frequency techniques", IEEE Trans. on Dielectrics and Electrical Insulation, vol. 18, no. 2, pp. 444 - 455, April 2011.

2. I. E. Portugués, P. J. Moore and I. A. Glover, "RF-based partial discharge early warning system for air-insulated substations," IEEE Trans. on Power Delivery, vol. 24, no. 1, pp. 20 - 29, January 2009.

3. P. J. Moore, I. Portugues and I. A. Glover, "Radiometric location of partial discharge sources on energized high-voltage plant", IEEE Trans. in Power Delivery, vol. 20, no. 3, pp. 2264-2272, July 2005.

4. J. M. S. de Souza Neto, E. C. T. de Macedo, T. C. M. Calvalcanti, E. C. Guedes, J. S. da Rocha Neto and I. A. Glover, "Development of a partial discharge emulator for calibration of a radiometric PD detection system," IEEE Int. Instrumentation and Measurement Technology Conf., Graz, Austria, May 2012.

5. T. Pinpart, M. D. Judd, "Differentiating between partial discharge sources using envelope comparison of ultra-highfrequency signals,” IET Science, Measurement and Technology, vol.4, no.5, pp.256-267, July 2009. 